\title{
Psychometric properties of the Beliefs about Emotions Scale in a normal population
}

\author{
Sohrab Amiri ${ }^{1}$, Abolghasem Yaghobi ${ }^{2}$ \\ ${ }^{1}$ Urmia University, Urmia, Iran \\ 2Bu-Ali Sina University, Hamedan, Iran \\ Neuropsychiatria i Neuropsychologia 2018; 13, 3: 85-90
}

Address for correspondence:

Sohrab Amiri, PhD

Urmia University

Urmia, Iran

e-mail: amirysohrab@yahoo.com

\begin{abstract}
Introduction: This study aimed to evaluate the psychometric properties of the Beliefs about Emotions Scale (BES) in the general population.

Material and methods: For this purpose, 558 (first study) and 120 (second study) subjects from a normal population were selected, then BES, Difficulties in Emotion Regulation Scale (DERS), Positive and Negative Affect Schedule (PANAS), Affect Intensity Measure (AIM), and Emotion Regulation Questionnaire (ERQ) questionnaires were distributed among them to respond. After collecting data, the reliability of the inventory was assessed by Cronbach's $\alpha$, retest, and split-half coefficient, then the criterion validity with other questionnaires was assessed to determine the psychometric properties of the BES. The factor structure was assessed by confirmatory factor analysis.

Results: The results of the factor analysis indicated that the BES has three factors, and checking the validity of the inventory using Cronbach's $\alpha$, retest, and split-half coefficient reflects the stability of the scale; the criterion validity of BES with other questionnaires showed desirable discriminant and convergence validity.

Conclusions: Overall, the findings indicated that BES has good psychometric properties in a normal population, and the tool can be used in studies in a normal population. However, it seems that BES has a different factor structure in a normal population.
\end{abstract}

Key words: negative emotions, factor structure, validity, reliability.

\section{Introduction}

Emotional disturbance is at the heart of many forms of psychopathology (Dillon et al. 2011; Kring 2008). Accordingly, beliefs about the unacceptability of experiencing negative emotions, or the adverse consequences of expressing such feelings, have been reported in individuals with a range of different problems (Surawy et al. 1995; Ali et al. 2000; Woolfolk and Allen 2007; Corstorphine 2006; Clark and Wells 1995; Jack 1991; Cramer et al. 2005).

Such beliefs may contribute to difficulties in a range of ways. For example, these beliefs may lead to unwanted feelings being ignored, which could retard the development of self-awareness and self-understanding, and hence the ability to look after oneself appropriately (Kennedy-Moore and Watson 2001). It has also been suggested that in individuals who are already emotionally vulnerable, inhibiting one's feelings can contrib- ute to feelings of numbness, emptiness, and an absence of a strong sense of identity (Linehan 1993). Furthermore, believing that it is unacceptable to express one's feelings is also likely to cause interpersonal problems; for example, by causing relationship difficulties to remain unresolved (Linehan 1993).

Believing that the expression of emotions or distress will be evaluated negatively by others can lead to safety-seeking behaviours to try to prevent this feared outcome, which can inadvertently maintain unhelpful beliefs and distress. For example, people who are concerned about appearing anxious can use strategies to try to hide their anxiety such as avoidance of eye contact, saying very little, or over-rehearsing speech, which can actually increase their social anxiety and/or impair social performance (Clark and Wells 1995). People who are feeling depressed may attempt to hide their low mood from others by avoiding social interaction, but 
Table 1. The research participants' descriptive and demographic characteristics

\begin{tabular}{|c|c|c|c|c|c|c|c|}
\hline \multicolumn{2}{|c|}{ Marital status } & \multirow{2}{*}{$\begin{array}{c}\text { Age } \\
\text { Mean (SD) }\end{array}$} & \multirow{2}{*}{ Percentage } & \multirow{2}{*}{ Number } & \multirow{2}{*}{ Group } & \multirow{2}{*}{ Aim } & \\
\hline Single (\%) & Married (\%) & & & & & & \\
\hline 93.3 & 6.7 & $27.8(3.6)$ & 57.4 & 342 & Women & Factor analysis, validity, & Study 1 \\
\hline 88.4 & 11.6 & $22.4(4.8)$ & 42.6 & 216 & Men & reliability & \\
\hline 90.8 & 9.2 & $24.9(4.1)$ & 100 & 558 & Total & & \\
\hline 92.9 & 7.1 & $25.9(3.2)$ & 61.7 & 81 & Women & Test-retest & Study 2 \\
\hline 89.4 & 10.6 & $23.5(3.9)$ & 38.3 & 39 & Men & & \\
\hline 91.4 & 8.6 & $24.6(3.7)$ & 100 & 120 & Total & & \\
\hline
\end{tabular}

the resulting reduced opportunity for positive reinforcement can lead to even lower mood and passivity (Ferster 1973).

Negative beliefs about emotions are addressed in many different therapies. Most involve psycho-education and the expression of emotions, which may help to modify such unhelpful beliefs, but different forms of therapy also have specific methods to address these beliefs. Treatments with a central emphasis on emotion have become increasingly popular over the past decade, including Dialectical Behaviour Therapy (Linehan 1993) and the Unified Protocol (Ellard et al. 2010). Many of these treatments include psychoeducation about emotion, including teaching clients to question judgments about emotions, because they tend to perpetuate maladaptive behaviours (Linehan 1993).

Together, research and clinical wisdom suggest there is utility in identifying emotional beliefs that may predict emotion regulation processes and distress (e.g. negative affect, symptoms of depression, or anxiety). The Beliefs about Emotions Scale (BES) has demonstrated associations with various psychological dimensions (Rimes and Chalder 2010; Dennison et al. 2010). Although a host of self-report measures assess constructs related to emotional beliefs, it is surprising that no questionnaire to assess such beliefs can be identified in general and normal population in the existing literature. This study aimed at validating a measure of beliefs about experiencing and expressing negative thoughts and feelings (BES), and it examined the internal reliability and validity of the scale in a normal population.

\section{Material and methods}

The participants were 558 (first study) and 120 (second study) subjects from a normal population. In this way, in three months, the research data were collected from the normal population samples. The inclusion criterion were age 18 years or older. People with a psychotic disorders, problems with substance abuse, acute suicidal tendencies, insufficient language skills, or severe cognitive impairment were excluded. Of the total participants, 387 people had high school diploma level of education or less $(57.09 \%)$, 234 people were undergraduates $(34.51 \%)$, and 57 had a master's degree and higher (8.12\%). Descriptive and demographic characteristics of the participants are presented in Table 1.

\section{Measures}

\section{Beliefs about Emotions Scale}

This scale has 12 items. The item content was selected to represent the types of beliefs about the unacceptability of experiencing and expressing emotions that have been specified in clinical reports and cognitive models (Surawy et al. 1995; Corstorphine 2006). The instructions were as follows: "Please tick the column that best describes how you think. Please note that because people are different, there are no right or wrong answers to these statements" (Rimes and Chalder 2010).

\section{Difficulties in Emotion Regulation Scale}

The Difficulties in Emotion Regulation Scale (DERS) contains 36 items to assess six dimensions of self-regulatory difficulties: no acceptance of emotional responses (accept), difficulties engaging in goal directed behaviour (when upset; goals), impulse control difficulties (when upset; impulse), lack of emotional awareness (aware), limited access to effective emotion regulation strategies (strategies), and lack of emotional clarity (clarity). Gratz and Roemer (2004) reported high internal consistency of the total DERS $(\alpha=0.93)$, adequate internal consistency of all subscales $(\alpha$ 's $>0.8)$, and also adequate 4-8-week test-retest reliability of the total scale $(r=0.88)$. The 4-8-week test-retest reliability of the subscales ranged from $r=0.69$ (no acceptance subscale) to $r=0.80$ (clarity subscale). The authors further reported evidence for convergent and predictive validity. 


\section{Positive and Negative Affect Schedule}

Positive and negative affect were measured with the Positive and Negative Affect Schedule (PANAS; Watson et al. 1988). The PANAS has been used to assess positive and negative affect in both young and older adults (Kercher 1992), and has shown high reliability and validity as a measure (Crawford and Henry 2004). In the present study an $\alpha=0.87$ was observed for negative affect and $\alpha=0.85$ for positive affect.

\section{Affect Intensity Measure (AIM)}

The Affect Intensity Measure (AIM; Larson and Deiner 1987) is a 120-item measure that assesses the intensity and reactivity in which respondents typically experience positive and negative emotions. The 10-item subscale, AIM-N, designed to assess the intensity of negative emotional experiences, was used in this study. Larson and Deiner (1987) report a test-retest reliability of 0.81 for the AIM after a three-month interval. Internal consistency is high for the AIM $(0.90 \leq \alpha \leq 0.94)$ and construct validity has been demonstrated in a number of samples (Goldsmith and Walters 1989; Larson and Deiner 1987).

\section{Emotion Regulation Questionnaire (ERQ)}

This scale consists of 10 items and assesses individual differences in two emotion regulation strategies: expressive suppression and cognitive reappraisal. The scale shows good psychometric properties (Gross and John 2003). Gross and John (2003) reported Cronbach's $\alpha$ coefficients, ranging between 0.79 (for the Reappraisal subscale) and 0.73 (for the Suppression subscale). The three-month test-retest reliability was 0.69 for both scales. Factor analyses supported the two-factor, orthogonal factor structure of the measure.

\section{Method of collection and analysis of data}

In order to collect data, the researcher had a meeting with the participants and gave the required explanations and then distributed the BES questionnaire (Rimes and Chalder 2010), DERS (Gratz and Roemer 2004), PANAS (Watson et al. 1988), AIM (Larson and Deiner 1987), and the Gross and John 2003) among them.

\section{Results}

\section{Factor analysis}

In order to investigate the fit of the threefactor structure of BES (Rimes and Chalder 2010) in a normal population, confirmatory factor analysis by maximum likelihood method and LISREL software were used (Jöreskog and Sörbom 2006). The diagram of the path of the conformity factor analysis with its coefficients are shown in Figure 1, and the $\mathrm{T}$ index is presented in Table 2.

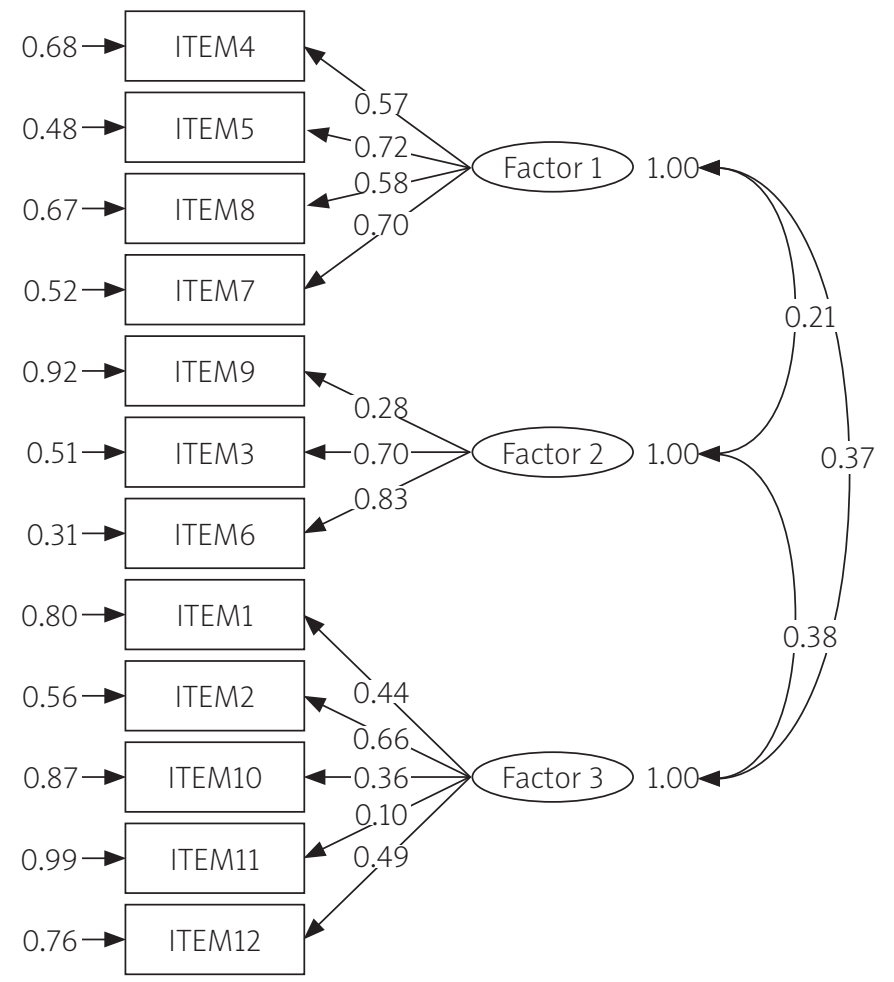

Fig. 1. Diagram of confirmatory factor analysis 
Table 2. T index of the Beliefs about Emotions Scale model

\begin{tabular}{lcccccccc} 
T index & Material & Subscale & T index & Material & Subscale & T index & Material & Subscale \\
5.1 & 1 & 3 & 7.6 & 3 & 2 & 7.6 & 4 & 1 \\
7.2 & 2 & & 8.3 & 6 & & 10.04 & 5 & 7 \\
4.1 & 10 & & 3.5 & 9 & & 9.6 & 8 \\
2.2 & 11 & & & & & & \\
5.7 & 12 & & & & & & \\
\hline
\end{tabular}

Table 3. The fit indices of the Beliefs about Emotions Scale model

\begin{tabular}{|c|c|c|c|c|c|c|c|c|c|c|c|c|}
\hline $\mathrm{CFI}$ & AGFI & GFI & IFI & RFI & NNFI & NFI & SRMR & RMSEA & $p$-value & $\mathrm{df}$ & $\chi^{2}$ & index \\
\hline 0.88 & 0.81 & 0.88 & 0.74 & 0.58 & 0.65 & 0.67 & 0.80 & 0.80 & $<0.05$ & 51 & 171.68 & value \\
\hline
\end{tabular}

CFI - comparative fit index, AGFI - adjusted goodness of fit index, GFI - goodness of fit index, IFI-incremental fit index, RFI-relative fit index, NNFI - non-normed fit index, NFI - normed fit index, SRMR - standardised root mean square residual, RMSEA - root mean square error of approximation

Table 4. The mean, standard deviation, $\alpha$, and test-retest coefficients of the Beliefs about Emotions Scale

\begin{tabular}{lcccc}
$\begin{array}{l}\text { Split-half coefficient } \\
(n=558)\end{array}$ & $\begin{array}{c}\text { Test-retest coefficient } \\
(n=120)\end{array}$ & $\begin{array}{c}\alpha \text { coefficient } \\
(n=558)\end{array}$ & Mean (SD) & Subscale \\
0.66 & $0.59^{*}$ & 0.72 & $15.8(3.1)$ & 1 \\
\hline 0.66 & $0.48^{*}$ & 0.68 & $9.3(5.7)$ & 2 \\
\hline 0.65 & $0.63^{*}$ & 0.76 & $15.6(5.4)$ & 3 \\
\hline
\end{tabular}

${ }^{*} p<0.01$

Table 5. Correlation coefficients between the subscales of Beliefs about Emotions Scale with other measures

\begin{tabular}{|c|c|c|c|c|c|c|c|c|c|}
\hline Suppression & Reappraisal & AIM & PANAS NA & PANAS PA & DERS & 3 & 2 & 1 & Subscale \\
\hline $0.14^{\star}$ & $-0.17^{\star}$ & $0.23^{\star *}$ & $0.33^{* *}$ & $-0.14^{*}$ & $0.31^{\star \star}$ & - & - & 1 & 1 \\
\hline 0.08 & $-0.12^{\star}$ & $0.26^{* *}$ & $0.36^{\star *}$ & $-0.16^{\star \star}$ & $0.36^{\star \star}$ & - & 1 & $0.23^{\star *}$ & 2 \\
\hline 0.10 & $-0.15^{\star}$ & $0.29^{* *}$ & $0.27^{* *}$ & $-0.21^{\star *}$ & $0.15^{\star}$ & 1 & $0.16^{\star}$ & $0.16^{*}$ & 3 \\
\hline
\end{tabular}

The confirmatory factor analysis using LISREL software achieved several fit index injectors shown in Table 3 (Brown 2006; Schermelleh-Engel et al. 2003; Hair et al. 2009; Hu and Bentler 1999; Tabachnick and Fidell 2007). The index with value of root mean square error of approximation $($ RMSEA $) \leq 0.8$ shows that the model is good ( $\mathrm{Hu}$ and Bentler 1999). The value of $\chi^{2} / \mathrm{df}$ was 3.3 , which is around 3, so the model's fit is acceptable. In addition, standardised root mean square residual $(\mathrm{SRMR})=0.08$ fit index showed the three-factor model's acceptable fit and comparative fit index $(\mathrm{CFI})=0.88$ and $\mathrm{RMSEA}=0.8$ showed the acceptable fit of the model.

\section{Validity, reliability}

To investigate the reliability of BES, Cronbach's $\alpha$, split-half, and test-retest coefficients were calculated. The 10-question scale's Cronbach's coefficient was 0.74 , which showed that the scale has good internal coordination. The split-half coefficient also indicated the high reliability of the scale and its subscale. A to- tal of 120 patients answered the questionnaire again four weeks later to calculate the retest coefficient, and the obtained scores' correlation coefficients were calculated after conduction of the three tests. The results of the Cronbach's $\alpha$, the split-half, and test-retest coefficients are presented in Table 4.

Table 4 shows that Cronbach's $\alpha$ coefficients were satisfactory and the data of all the test-retest and split-half s coefficients were significant. Therefore, it can be concluded that the BES has the desirable internal consistency in patients.

The validity of BES (Rimes and Chalder 2010) in a normal population was examined in three ways: the criterion validity was conducted simultaneously with the DERS (Gratz and Roemer 2004), PANAS (Watson et al. 1988), the AIM (Larson and Deiner 1987), the AIM (Gross and John 2003), and the correlation between subscales was determined. The results are shown in Table 5.

The pattern of correlation coefficients between the subscales in Table 3 shows that there is suit- 
able internal consistency between the subscales. The pattern of correlation coefficients between the subscales with the BES (Rimes and Chalder 2010), DERS (Gratz and Roemer 2004), PANAS (Watson et al. 1988), AIM (Larson and Deiner 1987), and the AIM (Gross and John 2003) indicates the concurrent criterion validity of the BES (Rimes and Chalder 2010) in a normal population.

\section{Conclusions}

The present study was done to evaluate and validate the BES in a normal population. The BES factor analysis showed that the three-factor solution has a good fit. This finding is inconsistent with the studies that examine the BES factor structure in chronic fatigue syndrome (CFS) (Rimes and Chalder 2010). The findings also indicated that the three factors have a desirable internal reliability, compared to the single-factor structure of the original questionnaire. Studying the BES factor structure and patterns of factor loadings, using confirmatory factor analysis, gave different results to those of Rimes and Chalder (2010), obtained by three-factor solution. All the factor loadings were higher than 0.4.

Investigation of the BES reliability, using $\alpha$ coefficients, test-retest coefficients, and split-half showed the scale's appropriate reliability. The subscale's $\alpha$ coefficients were, respectively, 0.72 , 0.68 , and 0.76 , and the amplitude of test-retest and split-half s coefficients suggested the BES's suitable reliability to measure beliefs about the unacceptability of experiencing or expressing negative emotions in a normal population. The findings are consistent with the study of Rimes and Chalder (2010), who designed the original measure of BES with chronic fatigue syndrome (CFS). However, the results indicate acceptable internal consistency. Perhaps with some changes in the questionnaire, a better questionnaire could be constructed for other samples, especially in clinical samples.

The validity of the BES with the DERS (Gratz and Roemer 2004), PANAS (Watson et al. 1988), AIM (Larson and Deiner 1987), AIM (Gross and John 2003), and their correlation, was significant, and it shows that the BES can be a good scale for measuring aspects of beliefs about emotions.

This result demonstrated the divergent validity of the BES. The BES's psychometric properties in the present study in a normal Population were respectively consistent with the studies done in the original version (Rimes and Chalder 2010), except that three subscales were indi- cated. According to what was said, the lack of a concise but valid and useful tool to assess the BES is the weakness of the research in this field. As a result, it seems that, regardless of language and culture, and considering the pattern of factor loadings, the BES is a useful tool to measure the fundamental structures related to emotional beliefs. But it seems that in clinical samples more care should be taken. In total, the psychometric properties of the BES is broadly applicable and has the capacity to measure emotional beliefs associated with a variety of disorders, and it can be also used in clinical and normal levels.

In summary, the reliability and validity analysis and confirmatory analysis demonstrated the desirable psychometric characteristics of the BES, and the present study's findings are consistent with the original version's (Rimes and Chalder 2010). The BES in normal population samples showed that it is a valid tool for assessing beliefs about the unacceptability of experiencing or expressing negative emotions. The calculated indices to evaluate the fit of the BES's model support the three-factor model's fit. So, according to what was said, the present study was conducted among normal population samples, and because it did not cover all the other groups, the results should be treated with caution in generalising. It is also suggested that future studies examine the BES's validity by using other psychological ways on the clinical population. The results of the present study indicate that the BES has acceptable validity and reliability in normal population samples. In addition, the questionnaire's factor structure was compliant with the designers' theory.

\section{References}

1. Ali A, Toner BB, Stuckless N, et al. Emotional abuse, self-blame, and self-silencing in women with irritable bowel syndrome. Psychosom Med 2000; 62: 76-82.

2. Brown TA. Confirmatory factor analysis for applied research. Guilford Press, New York 2006.

3. Clark DM, Wells A. A cognitive model of social phobia. In: Social phobia: diagnosis, assessment and treatment, Heimberg RG, Liebowitz MR, Hope DA, Schneier FR (Eds.). Guilford Press, New York 1995; 69-93.

4. Corstorphine E. Cognitive-emotional-behavioural therapy for the eating disorders: working with beliefs about emotions. Europ Eat Dis Rev 2006; 14: 448-461.

5. Cramer KM, Gallant MD, Langlois MW. Self-silencing and depression in women and men: Comparative structural equation models. Pers Ind Diff 2005; 39: 581-592.

6. Crawford JR, Henry JD. The Positive and Negative Affect Schedule (PANAS): construct validity, measurement properties and normative data in a large non-clinical sample. Br J Clin Psychol 2004; 43: 245-265. 
7. Dennison L, Moss-Morris R, Silber E, et al. Cognitive and behavioral correlates of different domains of psychological adjustment in early-stage multiple sclerosis. J Psychosom Res 2010; 69: 353-361.

8. Dillon DG, Deveney CM, Pizzagalli DA. From basic processes to real-world problems: How research on emotion and emotion regulation can inform understanding of psychopathology, and vice versa. Emot Rev 2011; 3: 74-82.

9. Ellard KK, Fairholme CP, Boisseau CL, et al. Unified protocol for the transdiagnostic treatment of emotional disorders: Protocol development and initial outcome data. Cogn Behav Pract 2010; 17: 88-101.

10. Ferster CB. A functional analysis of depression. Am Psychol 1973; 28: 857-870.

11. Goldsmith, RE, Walters H. A validity study of the Affect Intensity Measure. J Soc Behav Pers 1989; 4: 133-140.

12. Gratz KL, Roemer L. Multidimensional assessment of emotion regulation and dysregulation: development, factor structure, and initial validation of the difficulties in emotion regulation scale. J Psychopathol Behav Assess 2004; 26: 41-54.

13. Gross JJ, John OP. Individual differences in two emotion regulation processes: implications for affect, relationships, and well-being. J Pers Soc Psychol 2003; 85: 348362.

14. Hair JF, Black WC, Babin BJ, Anderson RE. Multivariate data analysis. Prentice Hall, 2009.

15. Hu L-T, Bentler PM. Cutoff criteria for fit indexes in covariance structure analysis: Conventional criteria versus new alternatives. Struct Equ Modeling 1999; 6: 1-55.

16. Jack DC. Silencing the self: women and depression. $\mathrm{Ha}$ rvard University Press, Cambridge 1991.

17. Jöreskog KG, Sörbom D. LISREL 8.80 for Windows [Computer Software]. Scientific Software International, Inc., Lincolnwood 2006.

18. Kennedy-Moore E, Watson JC. How and when does emotional expression help? Rev Gen Psychol 2001; 5: 187-212.

19. Kercher K. Assessing subjective well-being in the old-old: the PANAS as a measure of orthogonal dimensions of positive and negative affect. Res Aging 1992; 14: 131-167.

20. Kring AM. Emotion disturbances as transdiagnostic processes in psychopathology. In: Handbook of emotions. 3rd ed., Lewis L, Haviland-Jones JM, Barrett LF (Eds.). Guilford Press, New York 2008; 691-705.

21. Larson RJ, Deiner E. Affect intensity as an individual difference characteristic: A review. J Res Pers 1987; 21: 1-39.

22. Linehan M. Cognitive-behavioural treatment of borderline personality disorders. Guilford Press, New York 1993.

23. Rimes KA, Chalder T. The Beliefs about Emotions Scale: Validity, reliability and sensitivity to change. J Psychosom Res 2010; 68: 285-292.

24. Schermelleh-Engel K, Moosbrugger H, Müller H. Evaluating the fit of structural equation models: Tests of significance and descriptive goodness of fit measures. Psychol Res 2003; 8: 23-74.

25. Surawy C, Hackmann A, Hawton K, Sharpe M. Chronic fatigue syndrome: a cognitive approach. Behav Res Ther 1995; 33: 535-544.

26. Tabachnick BG, Fidell LS. Using multivariate statistics. Allyn \& Bacon, Inc. Needham Heights, MA, USA 2007.

27. Watson DA, Clark LA, Tellegen A (1988). Development and validation of brief measures of positive and negative affect: the PANAS scales. J Pers Soc Psychol 1988; 54: 1063-1070

28. Woolfolk RL, Allen LA. Treating somatization: a cognitive-behavioural approach. Guilford Press, New York 2007. 